\title{
The MHD Alfven wave oscillation model of kHz Quasi Periodic Oscillations of Accreting X-ray binaries
}

\author{
C. Zhang \\ 1 National Astronomical Observatories, Chinese Academy of Sciences, Beijing 100012, PR China \\ e-mail: zhangcm@bao.ac.cn \\ 2 Research Center for Theoretical Astrophysics, School of Physics, The University of Sydney, NSW 2006, Australia
}

Received 4 December 2003 / Accepted 19 April 2004

\begin{abstract}
We ascribe the interpretation of the twin kilohertz Quasi Periodic Oscillations (kHz QPOs) of X-ray spectra of Low Mass X-Ray Binaries (LMXBs) to MHD Alfvén wave oscillations in the different mass density regions of the accreted matter at the preferred radius, and the upper $\mathrm{kHz}$ QPO frequency coincides with the Keplerian frequency. The proposed model concludes that the $\mathrm{kHz}$ QPO frequencies depend inversely on the preferred radius, and that theoretical relation between the upper frequency $\left(v_{2}\right)$ and the lower frequency $\left(v_{1}\right)$ is $v_{1} \sim v_{2}^{2}$, which is similar to the measured empirical relation. The separation between the twin frequencies decreases (increases) with increasing $\mathrm{kHz}$ QPO frequency if the lower $\mathrm{kHz}$ QPO frequency is more (less) than $\sim 400 \mathrm{~Hz}$.
\end{abstract}

Key words. stars: neutron - X-rays: stars

\section{Introduction}

The launch of the X-ray timing satellite, Rossi X-ray Timing Explorer (RXTE), led to the discovery of Quasi Periodic Oscillations (QPOs) of LMXBs in their X-ray brightness, with frequencies $\sim 10^{-1}-10^{3} \mathrm{~Hz}$ (see van der Klis 2000 , for a recent review). Thereafter, much attention has been paid to the $\mathrm{kHz}$ QPO mechanism of LMXB; however the proposed models are still far from explaining all detected data. The $\mathrm{Z}$ sources (Atoll sources), which are high (less) luminous neutron star (NS) LMXBs (Hasinger \& van der Klis 1989), typically show four distinct types of QPOs (van der Klis 2000). At present, these are the normal branch oscillation (NBO) $v_{\mathrm{NBO}} \simeq 5-20 \mathrm{~Hz}$, the horizontal branch oscillation $(\mathrm{HBO})$ $v_{\mathrm{HBO}} \simeq 10-70 \mathrm{~Hz}$, and the kHz QPOs $v_{2}\left(v_{1}\right) \simeq 300-1300 \mathrm{~Hz}$ that typically occur in pairs in more than 20 sources, with upper frequency $v_{2}$ and lower frequency $v_{1}$. In 11 sources, nearly coherent burst oscillations $v_{\text {burst }} \simeq 270-620 \mathrm{~Hz}$ have also been detected during thermonuclear type I X-ray bursts; these are considered to be the NS spin frequencies $v_{\mathrm{s}}$ or their first overtone (see, e.g., Strohmayer \& Bildsten 2003). Moreover, the existence of a third $\mathrm{kHz}$ QPO has also been reported in three lowluminosity sources (Jonker et al. 2000). All of these QPOs but the burst oscillations have centroid frequencies that increase with the inferred mass accretion rate $\dot{M}$. Furthermore, the frequencies $v_{2}$ and $v_{1}$, as well as the frequencies $v_{2}$ and $v_{\mathrm{HBO}}$, follow very similar relations in five $\mathrm{Z}$ sources, and the QPO frequencies of LMXBs and black hole candidates (BHC) have a tight and systematical correlation over three orders of magnitude in frequency (Psaltis et al. 1998, 1999; Belloni et al. 2002).

Various theoretical models have been proposed to account for the QPO phenomenon in X-ray binaries (for a review see, e.g., Psaltis 2000). In the early detection of RXTE, the upper $\mathrm{kHz}$ QPO $\left(v_{2}\right)$ was simply considered to originate from the Keplerian orbital frequency at the preferred radius, and the lower $\mathrm{kHz}$ QPO $\left(v_{1}\right)$ is attributed to the beat of this frequency with the stellar spin frequency $v_{\mathrm{s}}$ (Strohmayer et al. 1996; Miller et al. 1998). However, this beat model is inadequate, for the detected frequency separation $\left(\Delta v \equiv v_{2}-v_{1}\right)$ decreased systematically with instantaneous $\dot{M}$ (see, e.g., van der Klis 2000). Later on, general relativistic effects were invoked to account for kHz QPOs (Stella \& Vietri 1999; Stella et al. 1999; Psaltis \& Norman 2000), which can satisfactorily explain the variation in $\mathrm{kHz}$ QPO separation $\Delta v$. Moreover, the theory of epicyclic parametric resonance in relativistic accretion disks was proposed (Abramowicz et al. 2003), where the twin $\mathrm{kHz}$ QPOs occur at the frequency of meridional oscillation and the radial epicyclic frequency in the same orbit, which can explain the frequency ratio 3:2 detected in black hole candidates. Although many other feasible ideas have also proposed, such as the disk seismic model (Wagoner 1999), a two-oscillator model (Osherovich \& Titarchuk) and the photon bubble model (Klein et al. 1996), no model has yet explained satisfactorily all observed QPO phenomena of LMXBs until now.

In this paper, the MHD Alfvén wave oscillation model is proposed, and its predictions and comparisons with the well detected sample sources are shown in the figures. 


\section{The model}

The model's idea is traced to the analogies with magnetic waves in coronal oscillations in solar physics, where the MHD turbulence driven by Alfvén wave oscillations occurs in the magnetic loops of the Sun's coronal atmosphere (Roberts 2000). While the dynamical details of the mechanisms responsible for the $\mathrm{kHz}$ QPOs in LMXBs are still uncertain, it is convenient to imagine that the MHD Alfvén wave oscillations occur at a certain preferred radius, where a MHD tube loop may be formed to conduct the accreted matter to the polar cap of star. Nevertheless, it is assumed that this preferred radius is a critical or a transitional radius where the spherical accretion matter with low mass density is transferred into polar channel accretion with high mass density that follows the loop and accretes onto NS polar cap. Possibly, this critical transition may give rise to MHD turbulence so that much more energy is liberated than at other positions.

As a phenomenological prescription, we associate the twin $\mathrm{kHz}$ QPO frequencies with the Alfvén wave oscillation frequencies (AWOFs) at a preferred radius described in the appendix, where the AWOF with the spherical accretion mass density coincides with the Keplerian orbital frequency $v_{\mathrm{K}}$ and the AWOF with the polar accretion mass density corresponds to a lower frequency. As a preliminary investigation, we are not concerned with the actual mechanisms of producing the $\mathrm{kHz}$ QPOs in the X-ray fluxes of LMXBs (see, e.g., Miller et al. 1998; Psaltis 2000). Rather, our main purpose is to stress the consistence between the model and the measured $\mathrm{kHz}$ QPO data, and leave the arguments concerning the possibility of the mechanism to a later paper.

At the preferred radius $\mathrm{r}$, the Alfvén velocity is, $v_{\mathrm{A}}(r)=$ $\frac{B(r)}{\sqrt{4 \pi \rho}}$, where $B(r)=B_{\mathrm{s}}(R / r)^{3}$ and $B_{\mathrm{S}}$ are the dipole magnetic field strengths at radius $r$ and at the surface of star with radius $R$, respectively. Therefore, the AWOF $v_{\mathrm{A}}$ is

$v_{\mathrm{A}}(r)=\frac{v_{\mathrm{A}}(r)}{2 \pi r}=\frac{B_{\mathrm{s}}(R / r)^{3}}{2 \pi r} \sqrt{\frac{S v_{\mathrm{ff}}}{4 \pi \dot{M}}} \propto \sqrt{S}$,

where the mass density of the accreted matter, $\rho=\dot{M} /\left[S v_{\mathrm{ff}}\right]$ (Shapiro \& Teukolsky 1983) is applied with the free fall velocity $v_{\mathrm{ff}}=\sqrt{2 G M / r}=c \sqrt{R_{\mathrm{S}} / r}$, where $R_{\mathrm{s}}=2 G M / c^{2}$ is the Schwarzschild radius and can be expressed as $R_{\mathrm{s}} \simeq 3 m(\mathrm{~km})=$ $0.3 m(10 \mathrm{~km})$ with $m=\frac{M}{M_{\odot}}$, the NS mass in units of solar mass, and the area $S$ representing the spherical area $S_{r}$ or the polar cap area $S_{\mathrm{p}}$, respectively,

$S_{r}=4 \pi r^{2}$,

$S_{\mathrm{p}}=4 \pi R^{2}\left(1-\cos \theta_{\mathrm{c}}\right), \sin ^{2} \theta_{\mathrm{c}}=R / r \equiv X$,

where $\theta_{\mathrm{c}}$ is the open angle of the last field line to close at radius $r$. As an approximation, the polar cap area is usually written as $S_{\mathrm{p}}=\frac{2 \pi R^{3}}{r}$ if $R \ll r$ (Shapiro \& Teukolsky 1983). For simplicity, it is convenient to write the two areas by means of the scaled radius parameter $X \equiv R / r$, so we have,

$S_{r}=4 \pi R^{2} X^{-2}, S_{\mathrm{p}}=4 \pi R^{2}(1-\sqrt{1-X})$.

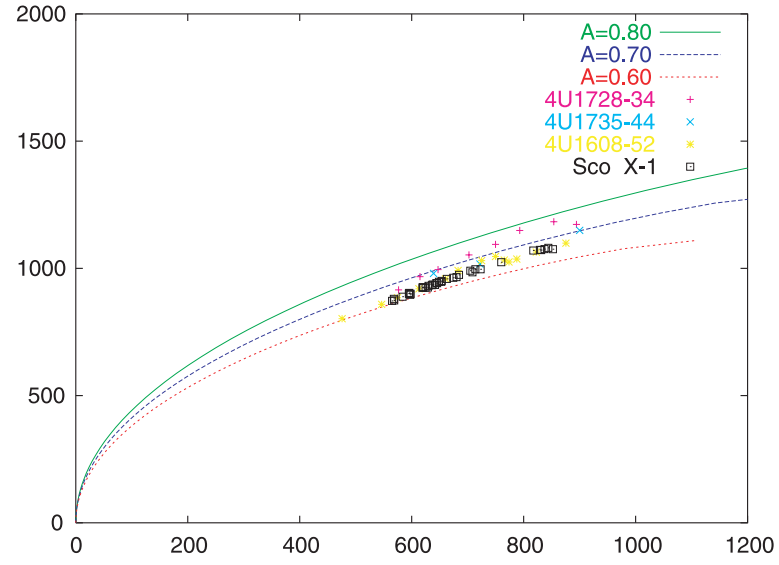

Fig. 1. $v_{2}$ versus $v_{1}$ plot. The horizontal axis is the lower $\mathrm{kHz}$ QPO frequency $v_{1}$ and the vertical axis is the upper $\mathrm{kHz}$ QPO frequency $v_{2}$. The kHz QPO data of four detected sample sources are plotted. The model presents a good consistence with the measured data for the NS averaged mass density parameters $A=0.6,0.7$ and 0.8 , which are shown in the three theoretical curves from bottom to top.

It is assumed that $v_{2}$ and $v_{1}$ are from the MHD Alfvén wave oscillations with the different accreted material mass densities, corresponding to the different areas $S_{r}$ and $S_{\mathrm{p}}$ respectively, and $v_{2}$ coincides with the Keplerian frequency at the preferred radius (see Appendix), therefore,

$v_{2}=\sqrt{\frac{G M}{4 \pi^{2} r^{3}}}=v_{\mathrm{A}}\left(S_{r}\right)=1850(\mathrm{~Hz}) A X^{3 / 2}$,

with the parameter $A$ defined as $A=\left(m / R_{6}^{3}\right)^{1 / 2}$ and $R_{6}=$ $R / 10^{6} \mathrm{~cm}$. The physical meaning of $A$ is clear in that $A^{2}$ represents the NS averaged mass density. So, by means of Eq. (1) with the correlation $v_{\mathrm{A}} \propto \sqrt{S}$, we obtain the lower $\mathrm{kHz}$ QPO frequency,

$v_{1}=v_{\mathrm{A}}\left(S_{\mathrm{p}}\right)=v_{2} \sqrt{S_{\mathrm{p}} / S_{r}}=v_{2} X \sqrt{1-\sqrt{1-X}}$.

The twin kHz QPO frequencies only depend on two variables, the parameter $A$ and the scaled radius $X$, so these two variables are implied if the twin $\mathrm{kHz}$ QPO frequencies are detected simultaneously. Therefore, the ratio of the twin frequencies is obtained easily, by setting $y(X)=\frac{v_{2}}{v_{1}}$,

$\frac{v_{1}}{v_{2}}=y^{-1}(X)=X \sqrt{1-\sqrt{1-X}}$

which is independent of the parameter $A$ and is only related to the parameter $X$. Furthermore, the twin frequency separation is given as follows,

$\Delta v \equiv v_{2}-v_{1}=v_{2}(1-X \sqrt{1-\sqrt{1-X}})$.

The comparisons of the model's conclusions to the four detected sample sources, Sco X-1 (van der Klis et al. 1997), 4U1608-52 (Méndez et al. 1998a,b), 4U1735-44 (Ford et al. 1998) and 4U1728-34 (Méndez \& van der Klis 1999), are shown in the figures. The relations $v_{2}$ versus $v_{1}$ and $\Delta v$ versus $v_{1}$ are plotted in Figs. 1 and 2, respectively, and they 


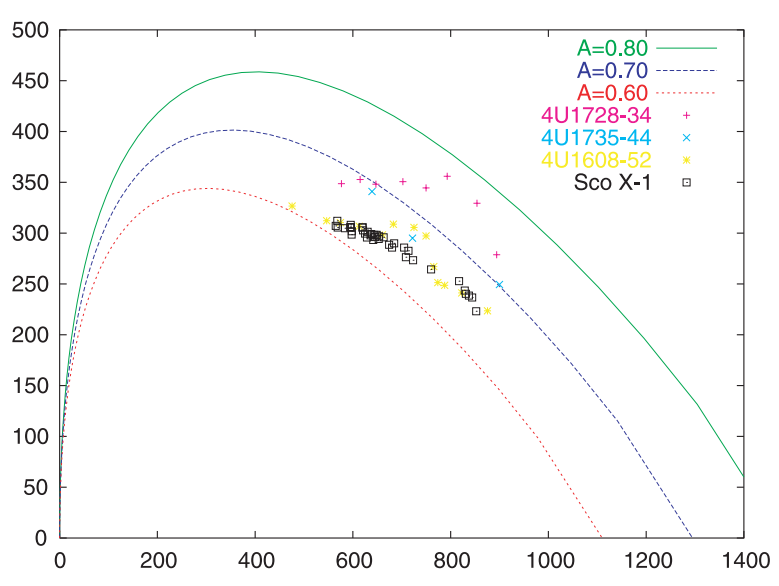

Fig. 2. $\Delta v$ versus $v_{1}$ plot. The horizontal axis is the lower $\mathrm{kHz}$ QPO frequency $v_{1}$ and the vertical axis is the twin $\mathrm{kHz}$ QPO frequency separation $\Delta v$. The kHz QPO data of four detected sample sources are plotted. The model presents a good consistence with the measured data for the NS averaged mass density parameters $A=0.6,0.7$ and 0.8 , which are shown in the three theoretical curves from bottom to top.

show that the agreement between the model and the observed QPO data is quite good for the selected ranges of NS parameters $A=0.6,0.7$ and 0.8. In Fig. 2, we find that $\Delta v$ increases with increasing $v_{1}$ if $v_{1}<408(A / 0.7) \mathrm{Hz}$ and $\Delta v$ decreases with increasing $v_{1}$ if $v_{1}>408(A / 0.7) \mathrm{Hz}$. The theoretical relation between the twin frequencies is derived from Eqs. (5) and (6),

$v_{1}=629(\mathrm{~Hz}) A^{-2 / 3} v_{2 k}^{5 / 3} \sqrt{1-\sqrt{1-\left(\frac{v_{2 k}}{1.85 A}\right)^{2 / 3}}}$,

where $v_{2 k}=v_{2} /(1 \mathrm{kHz})$. If $v_{2} \ll 1850 A(\mathrm{~Hz})=1295\left(\frac{A}{0.7}\right)(\mathrm{Hz})$, then we obtain the approximated theoretical relation between the twin $\mathrm{kHz}$ QPO frequencies,

$v_{1}=(382 / A)(\mathrm{Hz}) v_{2 k}^{2}=546(\mathrm{~Hz})\left(\frac{A}{0.7}\right)^{-1}\left(v_{2 k}\right)^{2}$.

A similar $v_{1}-v_{2}$ empirical correlation has also been found for the measured kHz QPO sources (see, e.g., Stella et al. 1999; Psaltis et al. 1998; Psaltis 2000; Psaltis \& Norman 2000). In Fig. 3, we plot the $v_{2} / v_{1}$ versus $v_{1}$ diagram, and it is found that the ratio $v_{2} / v_{1}$ decreases with increasing $v_{1}$. The averaged frequency ratio for the four sample sources is about $\langle y\rangle=1.4=7: 5$, corresponding to the averaged $X,\langle X\rangle=0.88$. In Fig. 4 , we plot $v_{2} / v_{1}$ versus $X$, and find that the theoretical curve is independent of the parameter $A$, which reflects the pure geometrical scaling relation between the twin frequency ratio and the parameter $X=R / r$. The $X$ distributions for the four examples are very similar (from $X=0.8$ to $X=0.93$ ), which implies that the dynamic mechanism that accounts for $\mathrm{kHz}$ QPO is intimately related to the scaled radius $X$ and has no direct relation with the other physical quantities.

In conclusion, the consistence between the model and the detections is robust, and the main results are summaried as follows: (1) the twin QPOs are inversely (proportionally) related to the radii (the accretion rate); (2) the theoretical relation between the twin frequencies is $v_{1} \sim v_{2}^{2}$; (3) the separation between the twin frequencies decreases (increases) with increasing the $\mathrm{kHz}$ QPO frequency if the lower $\mathrm{kHz}$ frequency is more

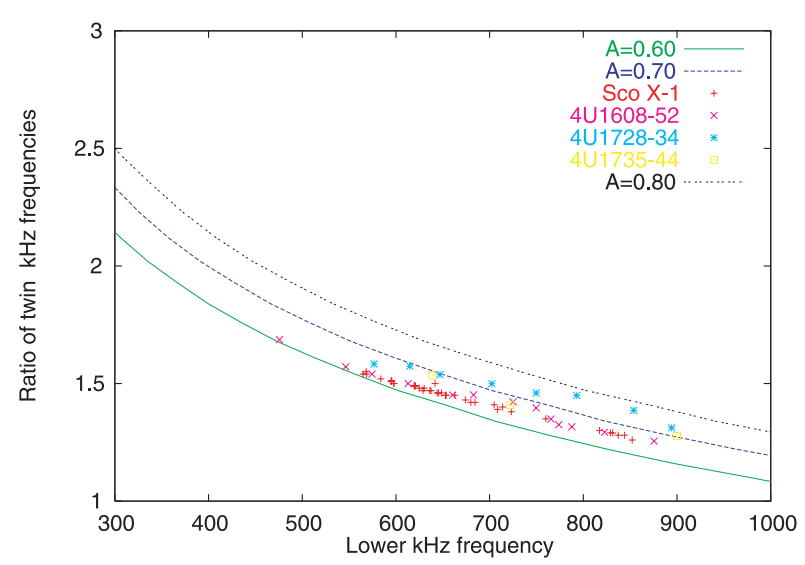

Fig. 3. The ratio between the twin $\mathrm{kHz}$ QPO frequencies versus the lower $\mathrm{kHz}$ QPO frequency. The three theoretical curves represent the NS mass density parameter conditions with $A=0.6,0.7$ and 0.8 from bottom to top. The data of four sample sources are plotted.

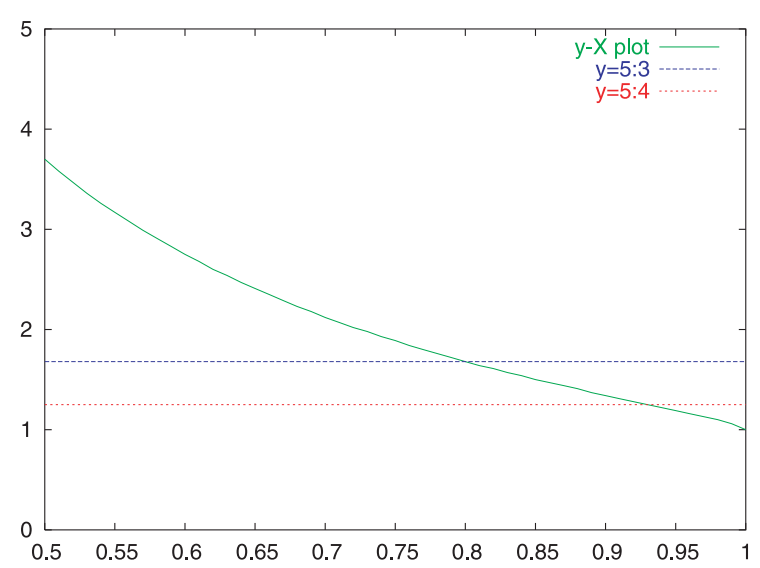

Fig. 4. The ratio between the twin $\mathrm{kHz}$ QPO frequencies versus the parameter $X \equiv R / r$. The horizontal axis is $X$ and the vertical axis is the ratio $y=v_{2} / v_{1}$. It is found that the theoretical curve is independent of the NS mass density parameter $A$. The two horizontal lines represent $y=5: 4$ and $y=5: 3$, from bottom to top, respectively, which cover the ratios of the detected $\mathrm{kHz}$ QPO data of the four samples.

(less) than $\sim 400 \mathrm{~Hz}$; (4) the ratio between the twin frequencies is only related to the scaled radius parameter $X$, and the homogenous $\mathrm{kHz}$ QPO frequency distributions of four detected sources indicate that these frequencies are from regions close to the surface of the NS, approximately, from $1.08 R$ to $1.25 R$. With regard to conclusion (3), for the theoretical $\Delta v-v_{1}$ relation with $v_{1}<400 \mathrm{~Hz}$ future detection is needed to confirm this prediction.

Acknowledgements. Thanks are due to T. Belloni, M. Méndez, and D. Psaltis for providing the data files, and discussion with T. P. Li is highly appreciated.

\section{Appendix: On the preferred radius}

The Alfvén radius $R_{\mathrm{A}}$ is determined by the condition that the magnetic ram pressure should match the plasma momentum energy density (Shapiro \& Teukolsky 1983), where $R_{\mathrm{A}}=1.9 \times 10^{6}(\mathrm{~cm}) B_{s 8}^{4 / 7} \dot{M}_{17}^{-2 / 7} m^{-1 / 7} R_{6}^{12 / 7}$, and $B_{\text {s8 }}$ the surface 
field in unit of $10^{8}$ Gauss, $\dot{M}_{17}$ the accretion rate in unit of $10^{17} \mathrm{~g} / \mathrm{s}$,

$\frac{B^{2}\left(R_{\mathrm{A}}\right)}{8 \pi}=\frac{1}{2} \rho\left(R_{\mathrm{A}}\right) v^{2}\left(R_{\mathrm{A}}\right)$,

or equivalently, $v_{\mathrm{A}}\left(R_{\mathrm{A}}\right)=\frac{B\left(R_{\mathrm{A}}\right)}{\sqrt{4 \pi \rho\left(R_{\mathrm{A}}\right)}}=v\left(R_{\mathrm{A}}\right)$, where $v_{\mathrm{A}}$ and $v$ represent the Alfvén velocity and the free fall velocity, $v(r)=$ $v_{\mathrm{ff}}(r)=\sqrt{2 G M / r}=c \sqrt{R_{\mathrm{s}} / r}=\sqrt{2} v_{k}(r)$ and $\rho(r)=\rho_{\mathrm{ff}}(r)=$ $\dot{M} /\left[S v_{\mathrm{ff}}(r)\right]$ with the area factor $S=4 \pi r^{2}$. In other words, the Alfvén velocity equals the free fall velocity at the Alfvén radius. If the area factor $S$ decreases (the polar cap area for instance), the mass density $\rho$ will increase and the Alfvén velocity will decrease. If we set $v(r)=v_{k}(r)$ and $\rho(r)=\rho_{\mathrm{ff}}(r)$, then we will obtain a preferred radius where the Alfvén velocity matches the Keplerian flow velocity,

$v_{\mathrm{A}}\left(R_{S 1}\right)=\frac{B\left(R_{S 1}\right)}{\sqrt{4 \pi \rho\left(R_{S 1}\right)}}=v_{k}\left(R_{S 1}\right)$.

So, the radius $R_{S 1}$ can be obtained to be $R_{S 1}=2^{2 / 7} R_{\mathrm{A}}=$ $1.22 R_{\mathrm{A}} \sim\left(B_{\mathrm{S}} / \dot{M}^{1 / 2}\right)^{4 / 7}$, where the Alfvén wave oscillation frequency matches the Keplerian frequency, $v_{\mathrm{A}}\left(R_{S 1}\right)=v_{\mathrm{K}}\left(R_{S 1}\right)$; we prefer to call it the quasi sonic-point radius as a distinction of the sonic-point radius by Miller et al. (1998) and Lai (1998).

\section{References}

Abramowicz, M. A., Bulik, T., Bursa, M., \& Kluzniak, W. 2003, A\&A, 404, L21

Belloni, T., Psaltis, D., \& van der Klis, M. 2002, ApJ, 572, 392

Ford, E. C., van der Klis, M., van Paradijs, J., et al. 1998, ApJ, 508, L155
Hasinger, G., \& van der Klis, M. 1989, A\&A, 225, 79

Jonker, P. G., Méndez, M., \& van der Klis, M. 2000, ApJ, 540, L29

Klein, R. L., Jernigan, G. J., Arons, J., Morgan, E. H., \& Zhang, W. 1996, ApJ, 469, L119

Lai, D. 1998, ApJ, 502, 721

Méndez, M., \& van der Klis, M. 1999, ApJ, 517, L51

Méndez, M., van der Klis, M., van Paradijs, J., Lewin, W. H. G., \& Vaughan, B. A. 1998a, ApJ, 494, L65

Méndez, M., van der Klis, M., Wijnands, R., et al. 1998b, ApJ, 505, L23

Miller, M. C., Lamb, F. K., \& Psaltis, D. 1998, ApJ, 508, 791

Osherovich, V., \& Titarchuk, L. 1999, ApJ, 522, L113

Psaltis, D. 2000, Adv. Space Res., 481, 281 [arXiv: astro-ph/0012251]

Psaltis, D., \& Norman, C. 2000 [arXiv:astro-ph/0001391]

Psaltis, D., Belloni, T., \& van der Klis, M. 1999, ApJ, 520, 262 [arXiv: astro-ph/9902130]

Psaltis, D., Méndez, M., Wijnands, R., et al. 1998, ApJ, 501, L95

Roberts, B. 2000, Sol. Phys., 193, 139

Shapiro, S. L., \& Teukolsky, S. A. 1983, Black holes, white dwarfs and neutron stars (New York: Wiley)

Stella, L., \& Vietri, M. 1999, Phys. Rev. Lett., 82, 17

Stella, L., Vietri, M., \& Morsink, S. 1999, ApJ, 524, L63

Strohmayer, T., \& Bildsten, L. 2003, in Compact stellar X-ray sources, ed. W. H. G. Lewin, \& M. van der Klis (Cambridge University Press) [arXiv: astro-ph/0301544]

Strohmayer, T., Zhang, W., Smale, A., et al. 1996, ApJ, 469, L9

van der Klis, M. 2000, ARA\&A, 38, 717

[arXiv: astro-ph/0001167]

van der Klis, M., Wijnands, A. D., Horne, K., \& Chen, W. 1997, ApJ, 481, L97

Wagoner, R. W. 1999, Phys. Rep., 311, 259

Zhang, W., Smale, A. P., Strohmayer, T. E., \& Swank, J. H. 1998, ApJ, 500, L171

Zhang, W., Strohmayer, T. E., \& Swank, J. H. 1997, ApJ, 482, L167 